\title{
Radio continuum observations of massive stars in open cluster NGC 6231 and the Sco OB1 association
}

\author{
Diah Y.A. Setia Gunawan ${ }^{1,2}$, Jessica M. Chapman ${ }^{1}$, Ian R. Stevens ${ }^{3}$, \\ Gregor Rauw ${ }^{4}$, and Claus Leitherer ${ }^{5}$ \\ ${ }^{1}$ CSIRO Australia Telescope National Facility, \\ PO Box 76, Epping, NSW 2121, Australia \\ ${ }^{2}$ Kapteyn Sterrenkundig Instituut, Rijksuniversiteit Groningen, \\ Postbus 800, 9700 AV Groningen, Nederland \\ ${ }^{3}$ School of Physics and Astronomy, University of Birmingham, \\ Edgbaston, Birmingham B15 2TT, UK \\ ${ }^{4}$ Institut d'Astrophysique et de Géophysique, Université de Liège, \\ 17 Allée du 6 août, B-4000 Liège 1 (Sart-Tilman), la Belgique \\ ${ }^{5}$ Space Telescope Science Institute ${ }^{1}$, \\ 3700 San Martin Drive, Baltimore, MD21218, USA
}

\begin{abstract}
We present results of the Australia Telescope Compact Array (ATCA) radio continuum observations of massive stars in the Sco OB1 association. Most stars detected in the program show spectral indices lower than value expected from thermal free-free emission.
\end{abstract}

\section{Introduction}

The Sco OB1 association, with the very young open cluster NGC6231 as its nucleus, stands out among $\mathrm{OB}$ associations because of its wealth of hot luminous stars. Therefore, at a relatively nearby distance of about $1.8 \mathrm{kpc}$, this region is ideal for studying the properties of the stellar winds from $\mathrm{OB}$ and Wolf-Rayet stars.

Radio observations of Wolf-Rayet and OB-type massive stars provide one of the key ways of studying their dense, ionized supersonic winds. The strong winds from such stars produce extended radio photospheres at $\mathrm{mm}$ and $\mathrm{cm}$ wavelengths, which result from optically-thick thermal free-free emission. At these wavebands, the thermal emission of continuous, spherically-symmetric winds are expected to have a spectral index of $\alpha \simeq 0.6$ (Wright \& Barlow 1975). In addition to the thermal emission, a significant fraction of early type stars show a non-thermal component. The origin of this is likely to be related to shock, either from colliding winds in a binary system (Eichler \& Usov 1993), or from chaotic winds of a single star (White 1985).

\section{Observation and results}

The six telescopes of the ATCA observed the Sco OB1 association at 3,6, 13 and $21 \mathrm{~cm}$ radio continuum wavebands (centred at $1384,2496,4800,8640 \mathrm{MHz}$, 


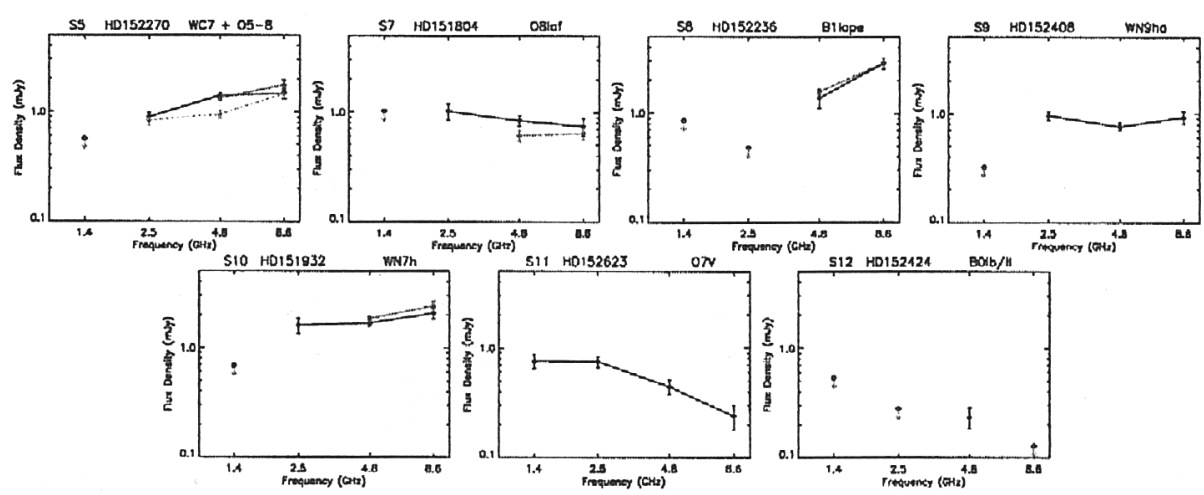

Figure 1. Flux-density of detected WR and OB stars.

Table 1. Radio spectral indices of WR and OB stars.

\begin{tabular}{|c|c|c|c|c|c|c|}
\hline no. & source & sp. type & $\alpha_{3-6 \mathrm{~cm}}$ & $\alpha_{6-13 \mathrm{~cm}}$ & $\alpha_{13-20 \mathrm{~cm}}$ & obs. date \\
\hline 5 & HD 152270 & $\mathrm{WC} 7+\mathrm{O} 5-8$ & $\begin{array}{l}0.41 \pm 0.08 \\
0.07 \pm 0.08 \\
0.65 \pm 0.09\end{array}$ & $\begin{array}{r}-\overline{0} \\
0.77 \pm 0.06 \\
0.21 \pm 0.08\end{array}$ & - & $\begin{array}{r}\text { March } 2000 \\
\text { October } 2000 \\
\text { July } 2001\end{array}$ \\
\hline 7 & HD 151804 & O8 Iaf & $\begin{array}{r}0.06 \pm 0.11 \\
-0.18 \pm 0.14\end{array}$ & $-0.33 \pm 0.12$ & - & $\begin{array}{r}\text { March 2000 } \\
\text { October 2000 }\end{array}$ \\
\hline 8 & HD 152236 & B1 Iape & $\begin{array}{l}0.94 \pm 0.08 \\
1.13 \pm 0.15\end{array}$ & - & - & $\begin{array}{r}\text { March } 2000 \\
\text { October } 2000\end{array}$ \\
\hline $\begin{array}{r}9 \\
10\end{array}$ & $\begin{array}{l}\text { HD } 152408 \\
\text { HD } 151932\end{array}$ & $\begin{array}{l}\text { WN9ha } \\
\text { WN7h }\end{array}$ & $\begin{array}{l}0.31 \pm 0.10 \\
0.41 \pm 0.08 \\
0.31 \pm 0.09\end{array}$ & $\begin{array}{r}-0.40 \pm 0.07 \\
-0.09 \pm 0.10\end{array}$ & - & $\begin{array}{c}\text { November } 2000 \\
\text { March } 2000 \\
\text { November } 2000\end{array}$ \\
\hline 11 & HD 152623 & O7 V & $-0.94 \pm 0.20$ & $-0.89 \pm 0.12$ & $0.02 \pm 0.11$ & November 2000 \\
\hline
\end{tabular}

respectively), during the period of 2000 March (3 and $6 \mathrm{~cm}$ only), October and November, and 2001 July. The observations, centred on 12 program stars in the association, were phase-referenced to $1740-517$, while the flux density level was calibrated to $1934-638$.

The data reduction was carried out using standard reduction procedure in the MIRIAD package. The images were subsequently CLEAN-ed from the effect of strong, extended extragalactic sources present in the field.

Seven out of 12 massive stars of Wolf-Rayet and OB type were detected at one or more wavelengths. Five of the six stars detected at two wavelengths or more show spectral indices that are lower than expected from thermal free-free emission.

\section{References}

Wright, A.E., Barlow, M.J. 1975, MNRAS 170, 41

Eichler, D., Usov, V. 1993, ApJ 402, 271

White, R.L. 1985, ApJ 289, 698 